\title{
Philosophy of Information: The Urgent Need to Move away from Entropy towards Algorithmic Information ${ }^{+}$
}

\author{
Hector Zenil ${ }^{1,2}$ \\ Department of Computer Science, University of Oxford, Oxford OX1 3BD, UK; hzenilc@gmail.com \\ 2 Department of Medicina Solna, Karolinska Institute, 17177 Solna, Sweden \\ + Presented at the IS4SI 2017 Summit DIGITALISATION FOR A SUSTAINABLE SOCIETY, Gothenburg, \\ Sweden, 12-16 June 2017.
}

Published: 9 June 2017

Discussions about information centered, either in favor or against, the use of Shannon's information theory are not only ill-suited but cannot help the discussion unless it is left behind just as the theory of randomness did early in the 1960s with the theory of algorithmic information. While it is true that physics, and many other areas, have been painfully slow at moving away from Shannon entropy and still can find a wide range of applications for it, we will show how eventually more powerful generating and predictive data-driven models will-and should-replace it. I will argue that discussions in Philosophy of Information should be thus one step ahead instead of several behind, guiding not only the philosophical discussion but also leading and steering scientific attention. I will explain the relevance of algorithmic complexity as a salient property at the core of the scientific method, especially in the challenge of causality discovery, and how fears against moving away from Entropy based upon arguments of uncomputability are unfounded, as it precludes progress and embraces defeat.

(C) 2017 by the author. Licensee MDPI, Basel, Switzerland. This article is an open access article distributed under the terms and conditions of the Creative Commons Attribution (CC BY) license (http://creativecommons.org/licenses/by/4.0/). 